\title{
Ionophore-Mediated Transmembrane Movement of Divalent Cations in Small Unilamellar Liposomes: An Evaluation of the Chlortetracycline Fluorescence Technique and Correlations with Black Lipid Membrane Studies
}

\author{
M.K. Mathew, R. Nagaraj, and P. Balaram \\ Molecular Biophysics Unit, Indian Institute of Science, Bangalore-560012, India
}

\begin{abstract}
Summary. Conceptual advances in the field of membrane transport have, in the main, utilized artificial membranes, both planar and vesicular. Systems of biological interest, viz., cells and organelles, resemble vesicles in size and geometry. Methods are, therefore, required to extend the results obtained with planar membranes to liposome systems. In this report we present an analysis of a fluorescence technique, using the divalent cation probe chlortetracycline, in small, unilamellar vesicles, for the study of divalent cation fluxes. An ion carrier (X537A) and a pore former (alamethicin) have been studied. The rate of rise of fluorescence signal and the transmembrane ion gradient have been related to transmembrane current and potential, respectively. A second power dependence of ion conduction - including the electrically silent portion thereof - on X537A concentration, has been observed. An exponential dependence of "current" on "transmembrane potential" in the case of alamethicin is also confirmed. Possible errors in the technique are discussed.
\end{abstract}

Key words Membrane-transport - fluorescence - chlortetracycline $\mathrm{X} 537 \mathrm{~A} \cdot$ alamethicin $\cdot$ ionophore $\cdot$ liposome

\section{Introduction}

Much of the conceptual advance in the field of membrane transport has been made with artificial membranes - planar (bimolecular lipid membranes, or BLM) and vesicular (liposomes) (Mueller, Rudin, Tien \& Wescott, 1962; Bangham, Standish \& Watkins, 1965: Mueller \& Rudin, 1967; Bean, Shepherd, Chan \& Eichner, 1969; Bangham, Hill \& Miller, 1974). The geometries of the two systems are complementary - the large surface-to-volume ratios of vesicles makes them ideally suited for measurements of net transmembrane solute transport while the BLM are preferred for studies of transmembrane potentials and transient ion conductances. Exchange kinetics in multilamellar liposomes are extremely complex (Johnson \& Bangham, 1969). Unilamellar vesicles, wherein a single bilayer membrane encloses an aqueous compartment, provide an excellent model system. However, the size of small, unilamellar vesicles (SUV) creates problems in studying solute influxes at constant gradient as, for a $300-\AA$ diameter vesicle, just 10 ions in the intravesicular space is of the order of $1 \mathrm{~mm}$ in the ion. Since the systems of interest - cells and organelles - have geometries and sizes better approximated by vesicles than by planar bilayers, methods for the extension of BLMtype studies to liposomes are required.

Of the available techniques for the measurement of intracellular potentials, the use of potential-sensitive dyes has been criticized on the basis of poor signal-to-noise ratios and specificity (Hladky \& Rink, 1976), while problems of sealing and electrolyte leakage complicate the microprobe electrode technique. Further, microprobe electrodes cannot be introduced into SUV. There remain, then, the techniques of estimating intracellular/vesicular solute concentrations - with the advantage of detecting electrically silent transport. Problems of time resolution preclude the use of radioactive tracer techniques, leaving optical probes as the most attractive alternative. In this report we present a fluorescence technique, using the divalent cation probe, chlortetracycline (CTC) (Caswell \& Warren, 1972; Mathew \& Balaram, 1980), in small, unilamellar liposomes, for the study of the ionophorous activities of an ion carrier (X537A) (Caswell \& Pressman, 1972) and a pore former (alamethicin) (Mueller, 1976) and relate the results so obtained with those of BLM experiments.

\section{Materials and Methods}

Egg phosphatidylcholine (EPC), X537A, CTC, Sephadex G-50, cholic acid and HEPES were from Sigma. Lanthanum chloride was obtained from Ventron. Alamethicin was synthesized as described earlier (Nagaraj \& Balaram, 1981). $\mathrm{CaCl}_{2}$ and $\mathrm{ZnCl}_{2}$ were of analytical grade. SUV were generated by the removal of cholate from mixed micelles of EPC and cholate by gel filtration on Sephadex G-50 as described by Brunner, Skrabal and Hauser (1976). The vesicles so generated have been reported to be uni- 


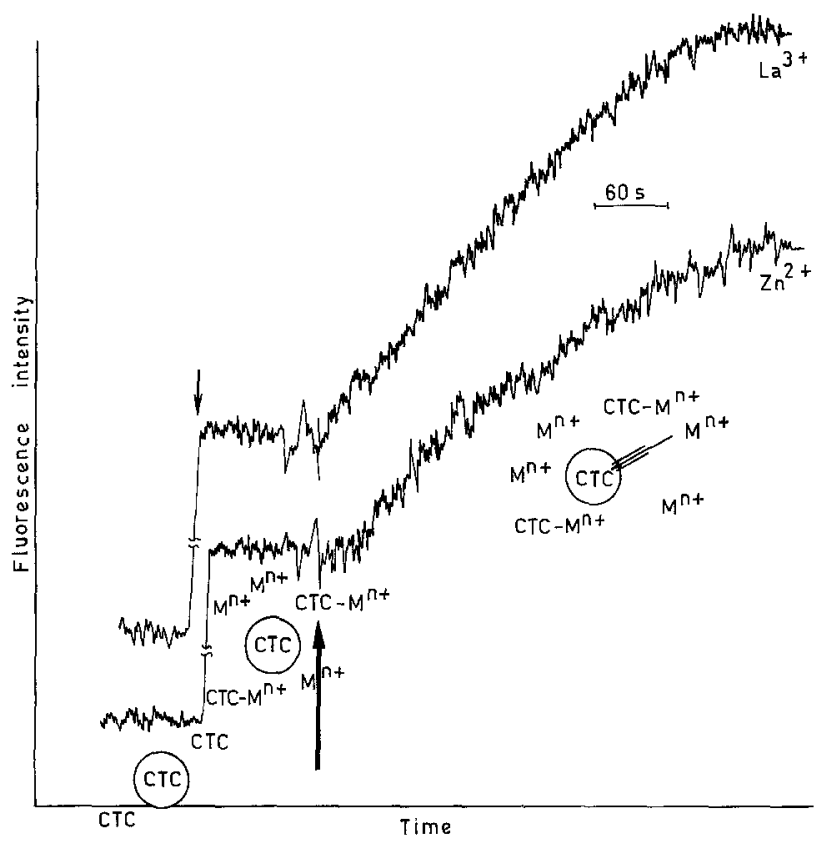

Fig. 1. Time-dependent increases in CTC-metal fluorescence on adding $5 \mu \mathrm{M}$ alamethicin. Cation concentrations used: $500 \mu \mathrm{M}$ $\mathrm{La}^{3+}, 1 \mathrm{mM} \mathrm{Zn}^{2+}$. Addition of test cation is indicated with small arrow. Addition of ionophore is indicated with thick arrow. The state of the system following each addition is schematically represented against the traces

lamellar with a narrow size distribution of mean diameter $300 \AA$. Ion transport was followed using $25 \mu \mathrm{M}$ CTC, $200 \mu \mathrm{g} / \mathrm{ml}$ vesicles in $5 \mathrm{~mm}$ HEPES, $100 \mathrm{~mm} \mathrm{NaCl}$, pH 7.0. After CTC had equilibrated across the membrane, the test cation was added at $1 \mathrm{~mm}$, or as stated, following which ionophore was introduced. The fluorescence of the CTC-metal complexes was monitored on a PerkinElmer model MPF-44A fluorescence spectrometer operated in the ratio mode with $10 \mathrm{~nm}$ excitation and emission band pass; $\lambda_{\mathrm{ex}}$ $=390 \mathrm{~nm}, \lambda_{\mathrm{em}}=530 \mathrm{~nm} .1-\mathrm{cm}$ path-length cells were used and solutions stirred with a magnetic pellet to minimize settling. Vesicle integrity was checked by a step response after adding the test cation followed by a sharp rise on adding $20 \mathrm{~m}$ X $537 \mathrm{~A}$.

\section{Results, Theory, and Discussion}

CTC is weakly fluorescent, whereas its complexes with $\mathrm{Ca}^{2+}, \mathrm{Mg}^{2+}, \mathrm{Zn}^{2+}$ and $\mathrm{La}^{3+}$ have a much higher quantum yield. The probe can thus be used to detect the presence of these cations. Our technique utilizes the ability of CTC to permeate lipid membranes, which remain impermeant to cations in the absence of ionophore. Equilibration of CTC across the membrane foiled attempts to entrap the probe in the vesicles, followed by removal of external CTC by gel filtration. Extravesicular CTC leads to a large basal fluorescence in the presence of test cations and necessitates extensive background suppression. Figure 1 shows the effect of adding ionophore to this suspension. The slow rise in fluores-
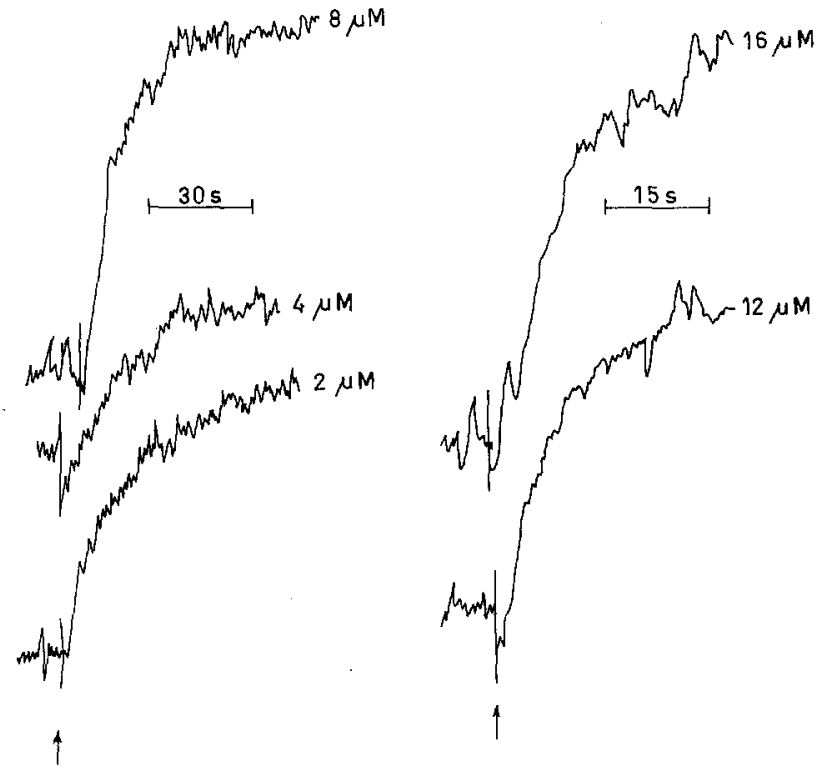

Fig. 2. Time-dependent increases in $\mathrm{CTC}-\mathrm{Ca}^{2+}$ fluorescence on adding $\mathrm{X} 537 \mathrm{~A}$. Concentrations of $\mathrm{X} 537 \mathrm{~A}$ are shown on figure. $\left[\mathrm{Ca}^{2+}\right]_{\mathrm{ext}}=1 \mathrm{~mm}$. Ionophore addition marked with an arrow

cence emission intensity is attributed to the entry of cation into the vesicles and binding to intravesicular CTC, the complex being highly fluorescent. This is effectively a charge translocation process.

i.e., $F \propto Q$

where $F$ is the incremental fluorescence intensity over the base line and $q$ the charge transported. It follows that

$d F / d t=K \cdot d q / d t=K I$

where $I$ is the transmembrane current and $K$ is a constant of proportionality. We are interested in studying ion translocation under conditions of specified gradient. With a vesicle diameter of $300 \AA$ (Brunner et al., 1976) and volume of $\sim 1.5$ $\times 10^{-20}$ liter, the gradient is rapidly broken down, the influx of just 10 ions per vesicle equalizing the concentrations across the membrane. We shall, therefore consider only the initial slope of the $F-t$ curve

$(d F / d t)_{0}=K I_{0}$.

We tested the dependence of ion translocating efficiency of X537A on ionophore concentration, under constant gradient conditions, for $\mathrm{Ca}^{2+}, \mathrm{Zn}^{2+}$ and $\mathrm{La}^{3+}$ as shown in Fig. 2. Célis, Estrada-O and 


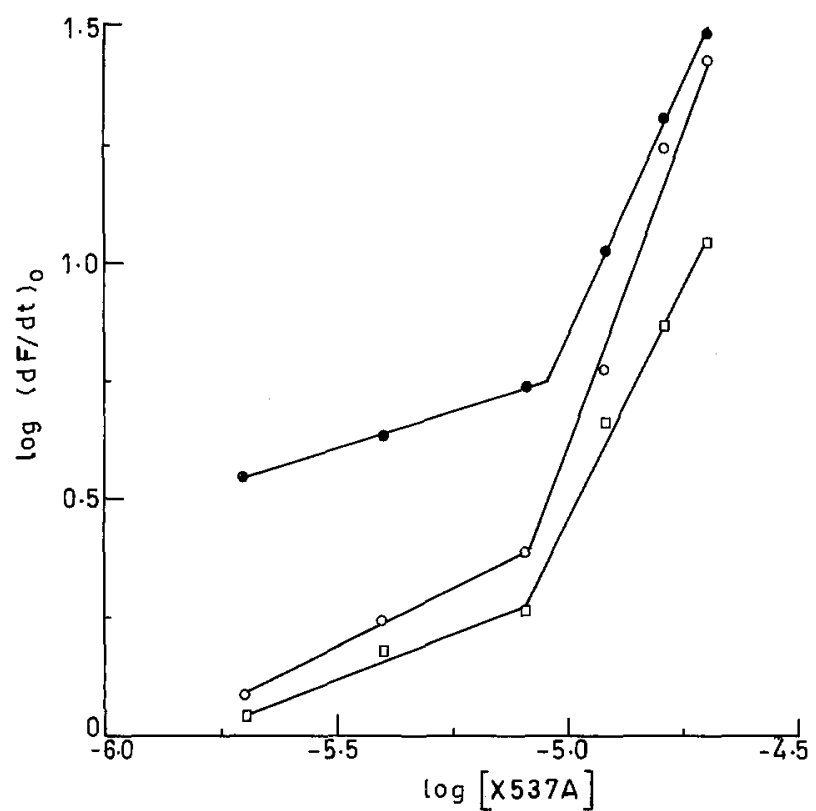

Fig. 3. Log-log plot of $(d F / d t)_{0}$ from Fig. 2 and similar experiments with $\mathrm{Zn}^{2+}$ and $\mathrm{La}^{3+}$ vs. X537A concentration. $\left[\mathrm{Ca}^{2+}\right]$, $1 \mathrm{mM} \mathrm{O}-0 ;\left[\mathrm{Zn}^{2+}\right], 1 \mathrm{mM} \square \longrightarrow ;\left[\mathrm{La}^{3+}\right], 500 \mu \mathrm{M} \bullet \longrightarrow$
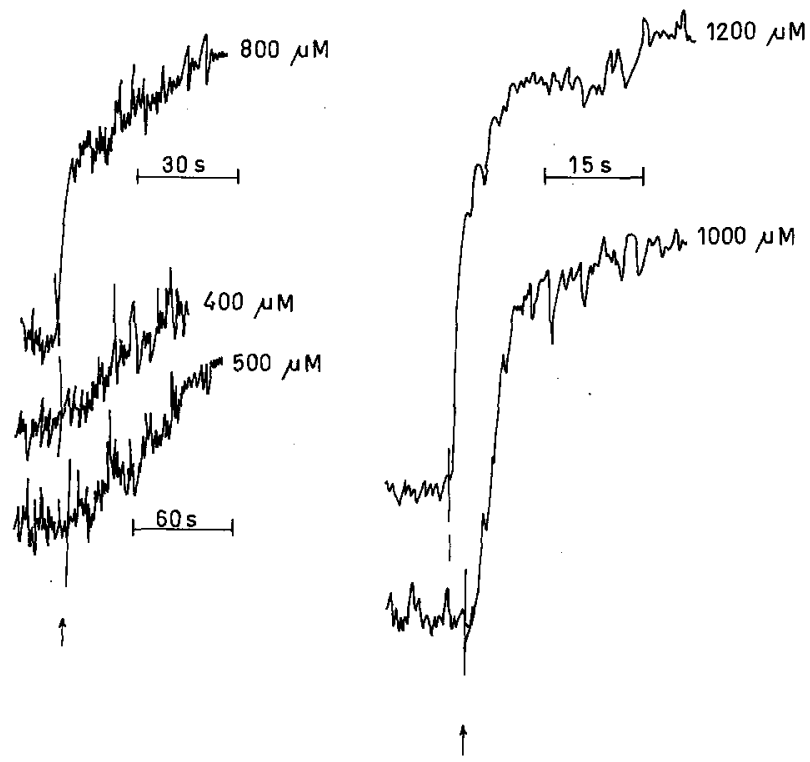

Fig. 4. Time-dependent increases in $\mathrm{CTC}-\mathrm{Ca}^{2}+$ fluorescence on adding $5 \mu \mathrm{M}$ alamethicin. $\left[\mathrm{Ca}^{2+}\right]_{\mathrm{ext}}$ concentrations are shown on figure. The upper time scale at left is for $800 \mu \mathrm{M}\left[\mathrm{Ca}^{2+}\right]_{\text {ext' }}$ the lower one for 400 and $500 \mu \mathrm{M}$. Time scale at right is for both 1000 and $1200 \mu \mathrm{M}\left[\mathrm{Ca}^{2+}\right]_{\mathrm{ext}}$. Ionophore addition is marked with an arrow

Montal (1974), in a similar experiment with BLM, obtained a sigmoidal curve in a log-log plot of membrane conductance $v s$. ionophore concentration. Since, at constant voltage, conductance is directly proportional to current, a similar curve should be obtained on plotting $\log (d F / d t)_{0}$ against $\log$ (X537A concentration). This is, indeed, obtained in Fig. 3. The differential response of CTC to the different cations (Caswell \& Hutchison, 1971; Mathew \& Balaram, 1980) has been taken into account by dividing the initial slopes $(d F / d t)_{0}$ by the relative intensities of the complexes $\left(F_{\mathrm{CTC}-M^{n+}}\right)$. The latter portions of the curves in Fig. 3 are linear with slopes around 2 for all three ions under study, in agreement with the results of Célis et al. (1974) for $\mathrm{Ca}^{2+}$. It is also in agreement with the results of Caswell and Pressman (1972), who proposed a 2:1 complex of $\mathrm{X} 537 \mathrm{~A}$ to metal, as the translocating unit. Note that the saturation of the curve reported by Célis et al. (1974) occurred only above $100 \mu \mathrm{M}$ X537A.

These authors obtained a linear dependence of conductance on calcium gradient and concluded that the translocating unit contained one $\mathrm{Ca}^{2+}$ ion. Pressman (1973), however, reports that the amount of $\mathrm{Ca}^{2+}$ transported by $\times 537 \mathrm{~A}$, as estimated by radioactive tracer techniques, is several orders of magnitude higher than that expected on the basis of electrical conductivity measurements - i.e., much of the transport is electrically silent. This is not entirely surprising as $\mathrm{H}^{+}$and $\mathrm{Na}^{+}$can both be transported by the ionophore, albeit less efficiently. Thus the net charge translocation detected represents only that fraction of the transport which is unaccompanied by the countertransport of $\mathrm{H}^{+}$and/or $\mathrm{Na}^{+}$. Our technique, which measures transmembrane ion fluxes directly, yields the same result of a second power dependence on X537A concentration for transport, indicating that the translocating unit does contain two X537A molecules, possibly analogous to the asymmetric sandwich structure of the $\mathrm{Ba}^{2+}$ complex of the ionophore (Johnson, Herrin, Lin \& Paul, 1970). The fact that charge translocation measurements arrive at the same conclusion could indicate that a constant proportion of the transport is electrogenic.

Changes in the transmembrane potential can be generated by varying the intra- and extra-vesicular ionic composition. It is expected that this potential should be approximately Nernstian (Sten-Knudsen, 1978), the concentrations of all permeant ions being considered. For alamethicin-modified membranes the distribution of all cationic species should be considered. Since the only asymmetrically distributed cation is $\mathrm{Ca}^{2+}$, only the contribution of $\mathrm{Ca}^{2+}$ need be taken into account. An exponential dependence of membrane current on applied voltage has been shown in the case of alamethicin (Eisenberg, Hall \& Mead, 1973). Thus, a log-log plot of 


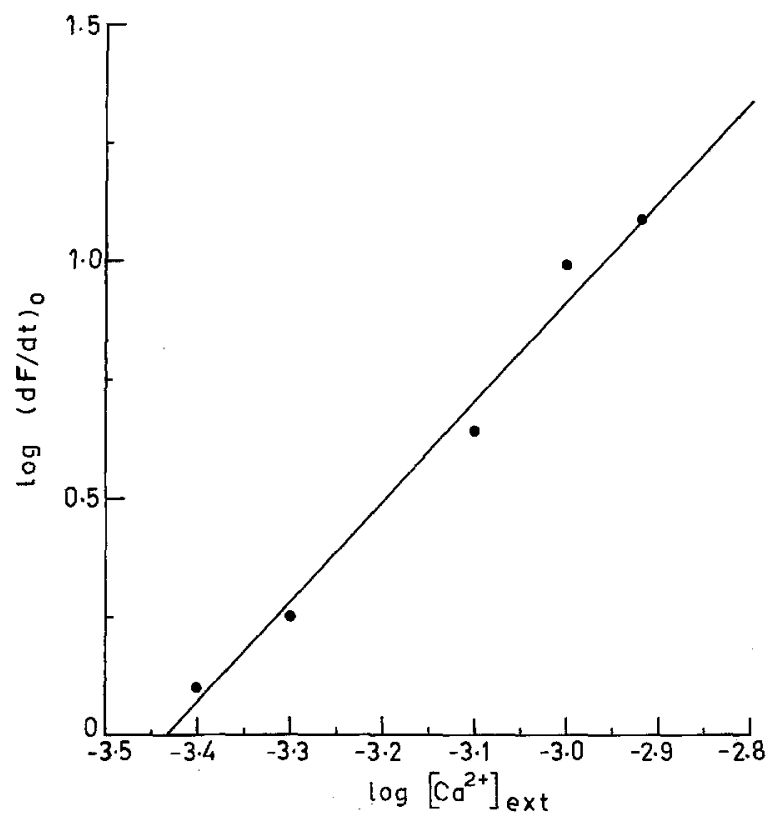

Fig. 5. Plot of $\log (d F / d t)_{0}$ from Fig. 4 vs. $\log \left[\mathrm{Ca}^{2+}\right]_{e x t}$

$(d F / d t)_{0} \quad v s$. external calcium concentration should be linear as the transmembrane potential will be given by

$V=V_{\text {salt }} \cdot \ln \left[M^{n+}\right] / C_{0}^{\prime}$

where $V_{\text {salt }}$ and $C_{0}^{\prime}$ are constants (Eisenberg et al., 1973) Fig. 4 shows the time-dependent fluorescence increase of $\mathrm{CTC}-\mathrm{Ca}^{2+}$ complexes in the presence of alamethicin, with varying concentrations of external calcium. Initial slopes of these curves are plotted against external calcium concentrations in a log-log plot in Fig. 5 and yields a straight line describable, in analogy with Eisenberg et al. (1973), as

$\log (d F / d t)_{0}=a+\log \left(\left[\mathrm{Ca}^{2+}\right]_{\mathrm{exl}} / b\right)$

where $a$ and $b$ are constants. It is important to note that no such correlation is obtained with the carrier X537A where exponential $I-V$ curves, in the steady state, have not been reported in BLM studies either.

These results confirm the analogy of $(d F / d t)_{0}$ with transmembrane current and of $\log \left[M^{n+}\right]_{\text {ext }}$ with the transmembrane potential, thus allowing the construction of steady state $I-V$ curves with liposomes. The severe size restrictions imposed by SUV can be relieved by the generation of larger unilamellar vesicles. Unilamellar liposomes of up to $1000 \AA$ can now be generated (Szoka \& Papahadjapoulos, 1980) to better approximate organelle dimensions. The estimation of the stoichiometry of the translocating units is relatively simple in the case of BLM where transport across only one membrane at a time is being studied. In the liposome case, distribu- tion of ionophore among vesicles has to be properly modelled. In the case of pre-formed aggregates entering the lipid phase, this is relatively simple, as for X537A. However, lipid phase aggregation to form oligometric aggregates, as has been proposed for alamethicin, is harder to model. It has been proposed that alamethicin enters the membrane as a monomer and subsequently undergoes reversible aggregation in the lipid phase (Mueller \& Rudin, 1968). Boheim and Kolb (1978) have proposed that alamethicin pre-aggregates enter the membrane, under the influence of an applied electric field. However, Fringeli (1980) and Fringeli and Fringeli (1979), using Attenuated Total Reflection IR spectroscopy conclude that the ionophore enters the lipid phase and subsequently aggregates in this phase even in the absence of any field. Furthermore, noise in the system is currently too high to detect single-channel conduction even if the number of vesicles were to allow the controlled formation of a limited number of channels.

There exist three possible errors in this technique. Firstly, since the membrane is permeable to CTC an error could be introduced by the probe migrating across the membrane and binding external cation leading to a rise in fluorescence independent of ionophore. Secondly, CTC has been reported to act as a ionophore in pancreatic islet cells at concentrations above $100 \mu \mathrm{M}$ (Sehlin \& Taeljedahl, 1979). Ionophorous activity is not, however, expected at the concentrations used in our experiment. We observe a step response on adding the test cation to the CTC-loaded vesicle suspension (Fig. 1) with no slow rise in fluorescence with time, indicating that no further complexation is occurring after the initial rise attributed to the complexation of the extravesicular CTC. Further, a rise in fluorescence on adding ionophore (which does not, of itself, enhance CTCmetal fluorescence) indicates a nonuniform distribution of test cations and uncomplexed CTC across the membrane prior to ionophore addition. Thirdly, the observed rise in fluorescence could occur due either to cations entering the liposome or CTC leaving it, under the influence of added inophore. $\mathrm{X} 537 \mathrm{~A}$ is known to transport biogenic amines (Schadt \& Haeusler, 1974) and CTC has a dimethylamino function. Transport of CTC by X537A is expected to be minimal as the ionophore complexes weakly with secondary and tertiary amines. The alamethicin pore is large enough to allow the passage of CTC or its complexes through it (Mueller, 1976). The fact, however, that good correlations have been obtained with a totally different technique, viz. BLM studies, indicates that such CTC fluxes are minimal during these experiments. 
The technique thus serves to bridge the extant technologies of BLM and liposome work, allowing the study of transmembrane ion fluxes in liposome systems. An application of this technique to evaluate the structural requirements for pore formation by alamethicin and its synthetic fragments has been reported (Nagaraj, Mathew \& Balaram, 1980).

\section{References}

Bangham, A.D., Hill, M.W., Miller, N.G.A. 1974. Preparation and use of liposomes as models of biological membranes. In: Methods in Membrane Biology. E.D. Korn, editor. Vol. 1, pp. 1-68, Plenum, New York

Bangham, A.D., Standish, N.M., Watkins, J.C. 1965. Diffusion of univalent jons across the lamellae of swollen phospholipids. $J$. Mol. Biol. 13:238-252

Bean, R.C., Shephered, W.C., Chan, H., Eichner, J. 1969. Discrete conductance fluctuations in lipid bilayer protein membranes. J. Gen. Physiol. 53:741-757

Boheim, G., Kolb, H.-A. 1978. Analysis of multi-pore systems of alamethicin in a lipid membrane. I. Voltage-jump currentrelaxation measurements. J. Membrane Biol. 38:99-150

Brunner, J., Skrabal, P., Hauser, H. 1976. Single bilayer vesicles prepared without sonication. Physico-chemical properties. Biochim. Biophys. Acta 455:322-331

Caswell, A.H., Hutchison, J.D. 1971. Selectivity of cation chela tion to tetracyclines: Evidence for special conformation of calcium chelate. Biochem. Biophys. Res. Commun. 43:625-630

Caswell, A.H., Pressman, B.C. 1972. Kinetics of transport across sarcoplasmic reticulum vesicles induced by ionophores. Bio chem. Biophys. Res. Commun. 49:292-298

Caswell, A.H., Warren, S. 1972. Observation of $\mathrm{Ca}^{2+}$ uptake by isolated sarcoplasmic reticulum employing a fluorescent chelate probe. Biochem. Biophys. Res. Commun. 46:1757-1763

Célis, H., Estrada-O., S., Montal, M. 1974. Model translocators for divalent and monovalent ion transport in phospholipid membranes. I. The ion permeability induces in lipid bilayers by the antibiotic X-537 A. J. Membrane Biol. 18:187-199

Eisenberg, M., Hali, J.E., Mead, C.A. 1973. The nature of the voltage-dependent conductance induced by alamethicin in black lipid membranes. J. Membrane Biol. 14:143-176

Fringeli, U.P. 1980. Distribution and diffusion of alamethicin in a lecithin/water model membrane system. J. Membrane Biol. 54:203-212

Fringeli, U.P., Fringeli, M. 1979. Pore formation in lipid membranes by alamethicin. Proc. Natl. Acad. Sci. USA 76:38523856
Hladky, S.B., Rink, T.F. 1976. Membrane potentials and properties of human erythrocytes and ghosts assessed with a fluorescent dye, 3,3'-dipropyl-2,2' thiadicarbocyanine. J. Physiol. (London) 258:100P

Johnson, S.M., Bangham, A.D. 1969. Potassium permeability of single compartment liposomes with and without valinomycin. Biochim. Biophys. Acta 193:82-91

Johnson, S.M., Herrin, J., Lin, S.J., Paul, I.C. 1970. The crystal and molecular structure of the barium salt of an antibiotic containing a high proportion of oxygen. J. Am. Chem. Soc. 92:4428-4435

Mathew, M.K., Balaram, P. 1980. A reinvestigation of chlortetracycline fluorescence: Effect of $\mathrm{pH}$, metal ions and environment. J. Inorg. Biochem. 13:339-346

Mueller, P. 1976. Molecular aspects of electrical excitation in lipid bilayers and cell membranes. In: Horizons in Biochemistry and Biophysics. E. Quagliariello, F. Palmieri, and T.P. Singer, editors. Vol. 2, pp. 230-284. Addison-Wesley, Reading (Mass.)

Mueller, P., Rudin, D.O. 1967. Development of $\mathrm{K}^{+}-\mathrm{Na}^{+}$discrimination in experimental bimolecular lipid membranes by macrocyclic antibiotics. Biochem. Biophys. Res. Commun. $26: 398-404$

Mueller, P., Rudin, D.O. 1968. Action potentials induced in bimolecular lipid membranes. Nature (London) 217:713-719

Mueller, P., Rudin, D.O., Tien, H.Ti., Wescott, W.C. 1962. Reconstitution of cell membrane structure in vitro and its transformation into an excitable system. Nature (London) 294:979980

Nagaraj, R., Balaram, P. 1981. Solution phase synthesis of alamethicin I. Tetrahedron 37:1263-1270

Nagaraj, R., Mathew, M.K., Balaram, P. 1980. Cation translocating effects of alamethicin and its synthetic fragments in lipid membranes. Influence of peptide chain length and charge. FEBS Lett. 121:365-368

Pressman, B.C. 1973. Properties of ionophores with broad range cation selectivity. Fed. Proc. 32:1698-1703

Schadt, M., Haeusler, G. 1974. Permeability of lipid bilayer membranes to biogenic amines and cations: Changes induced by ionophores and correlations with biological activities. $J$. Membrane Biol. 18:277-294

Sehlin, J., Taeljedahl, I.B. $1979 .{ }^{45} \mathrm{Ca}$ ion uptake by dispersed pancreatic islet cells: Effects of D-glucose and chlortetracycline. Pfluegers Arch 381:281-285

Sten-Knudsen, O. 1978. Passive transport processes. In: Membrane Transport in Biology. G. Giebisch, D.C. Tosteson, and H.H. Ussing, editors. Vol. 1, pp. 5-113. Springer-Verlag, Berlin

Szoka, F., Papahadjopoulos, D. 1980. Comparative properties and methods of preparation of lipid vesicles (liposomes). Annu. Rev. Biophys. Bioeng. 9:467-508

Received 13 October 1980 ; revised 21 May 1981 